ISSN: 2638-5279

Volume 3, Issue 2, 2020, PP: 10-17

\title{
Patient Risk Stratification and Demographic Profile based on Serum Vitamin-D Levels: A Cross-Sectional Observational Study
}

\author{
Mousumi Sanyal ${ }^{1}$, Richmond Ronald Gomes ${ }^{2}$, Yearul Kabir ${ }^{3}$, Sajib Chakraborty ${ }^{4}$
}

${ }^{1}$ Assistant Professor, Medicine, Ad-din Women's Medical College Hospital, Dhaka, Bangladesh.

${ }^{2}$ Associate Professor, Medicine, Ad-din Women's Medical College Hospital, Dhaka, Bangladesh.

${ }^{3}$ Professor, Department of Biochemistry and Molecular Biology, University of Dhaka.

${ }^{4}$ Associate Professor, Department of Biochemistry and Molecular Biology, University of Dhaka.

*Corresponding Author: Mousumi Sanyal, Assistant Professor, Medicine, Ad-din Women's Medical College Hospital, 2 Bara Maghbazar, Dhaka1217, Bangladesh.

\section{Abstract}

Background: Vitamin D deficiency is now recognized as a pandemic.An estimated 1 billion people worldwide, across all ethnicities and age groups, have a vitamin D deficiency (VDD). This pandemic of hypovitaminosis $D$ can mainly be attributed to lifestyle (for example, reduced outdoor activities) and environmental (for example, air pollution) factors that reduce exposure to sunlight. High prevalence of vitamin D deficiency is a particularly important public health issue because hypovitaminosis $D$ is an independent risk factor for total mortality in the general population. The currentstudy sought to uncover the population-wide true prevalence of Hypovitaminosis $D$ in Bangladesh by harnessing large amount patient data and subsequently analyze the vitamin D levels of the pre-categorized age and gender groups.

Methods: In this cross-sectional observational study, serum samples were collected from 7951 selected cases (including children and adult) who attended both indoor and outdoor department of a Private hospital in Dhaka, Bangladesh. Study period was from July 2017 to June 2018. Levels of 25(OH)D (in $\mathrm{ng} / \mathrm{ml}$ ) was done in every caseusing the chemiluminescent micro-particle immunoassay.

Results: $81.36 \%$ ( $n=6469$ ) of the study population was affected with Hypovitaminosis DOnly a small fraction of study subjects ( $n=58$ ) representing $0.7 \%$ of the total study-population was suffering from vitamin $D$ toxicity. Among the study subjects ( $n=6469)$ with Hypovitaminosis $D, 56.60 \%$ patients $(n=4501)$ were deficient in vitamin $D$ while the rest of the study subjects $-24.70 \%$ were insufficient $(n=1967)$. Highest female to male ratio was found for the deficient group (3.52) followed by the insufficient group (2.70) and normative group (2.44). The lowest female to male ratio was identified for toxic group (1.63).Regarding age impact, the predominance of Vitamin D deficiency compared of the study subjects with age from 20 to 60.For males,Vitamin D deficiency fraction is almost uniformly distributed ranging from $39 \%$ to $46 \%$ across all age group with the exception of the age group $\geq 80$ in which the fraction of the Vitamin D deficient subjects was $25 \%$. In contrast, for females, there was a large variation of in the number of Vitamin D deficient subjects. For instance, the highest fraction of Vitamin D deficient subjects were identified in the age group 10-19 (69\%) closely followed by 20-21 (68\%) and 30-39 (67\%). The later age groups - 40-49, 50-59, 60-69 and 70-79 showed a decreased fraction of Vitamin $D$ deficient subjects $-59 \%, 53 \%, 46 \%$ and $45 \%$ respectively.

Conclusion: As there is continuous increasing number of VDD patients, our findings strengthen the rationale for widespread vitamin D supplementation to prevent premature mortality, emphasize the need for it early in life and mitigate concerns about a possible negative effect at higher levels.

Keywords: Vitamin D deficiency(VDD),Hypovitaminosis D, pandemic. 
Patient Risk Stratification and Demographic Profile based on Serum Vitamin-D Levels: A Cross-Sectional Observational Study

\section{INTRODUCTION}

Vitamin D is known as sunshine vitamin. Vitamin D deficiency (VDD) is an emerging challenge in our daily clinical practice due to its increased incidence. Currently half of the world population are being affected by VDD1.Due to itsalarming increase in incidence, VDD is being considered among one of the pandemic non-communicable diseases2.The definition of Hypovitaminosis D includes both deficiency and insufficiency states. Different literature and different society has defined these two condition in different ways due to lack of current consensus regarding the cutoff value for the definition of vitamin deficiency3. Levels of 25(OH)D are interpreted as follows4:21-29 $\mathrm{ng} / \mathrm{mL}$ (52.5-72.5 nmol/L): Vitamin D insufficiency and $<20 \mathrm{ng} / \mathrm{mL}(<50 \mathrm{nmol} / \mathrm{L})$ : Vitamin D deficiency. This cutoff value is suitable most for tropical country like Bangladesh.In india, a similar tropical country like Bangladesh,The Endocrine Society of India defines 25(OH)D levels between 20 and $40 \mathrm{ng} / \mathrm{mL}$ (50-100 $\mathrm{nmol} / \mathrm{L}$ ) as adequate for most of the population5.Few literature recommends that, the serum $25(\mathrm{OH}) \mathrm{D}>30$ $\mathrm{ng} / \mathrm{mL}(75 \mathrm{nmol} / \mathrm{L})$ may provide additional health benefits than a cutoff above $20 \mathrm{ng} / \mathrm{mL}(50 \mathrm{nmol} / \mathrm{L})$ for individuals presenting with conditions such as osteoporosis, obesity, pregnancy, lactation, elderly, malabsorption syndromes, renal or liver insufficiency, and medications interfering with the Vitamin D metabolism5,6.

\section{Materials ANd Methods}

Thiscross-sectionalobservationalstudywasperformed on 7951 patients (including children \& adult) who attended both Outdoor and Indoor Departments of a Private Hospital in Dhaka, Bangladesh over the period of July 2017 to June 2018. All the patients presented with the suggestive clinical presentation, such as bodyache, muscle pain, fatigue, fibromyalgia, retarded growth in children. After collecting their vitamin D level, data were analyzed according to age and gender using powerful statistical tests/algorithm. To estimate the true prevalence, the number of subjects attended the private hospital in Dhaka within the same time frame (July 2017 to June 2018) was considered as the background population for normalization purpose.

\section{RESULTS}

To predict the occurrence of population wide hypovitaminosis $\mathrm{D}$, the most limiting factor is the sample size. To overcome this challenge and to strengthen the statistical power, the current study includes a large number of study-subjects $(n=7951)$ that were tested for serum vitamin D (25 hydroxy Vitamin D) levels during the period of one year (July 2017- June 2018). To stratify the study subjects according to their serum vitamin D levels, we took advantage of previously published data and categorize the study population into four groups: Deficient, Insufficient, normative and toxic. Both deficient and insufficient groups constitute Hypovitaminosis D group.

The analysis revealed that $81.36 \%(n=6469)$ of the study population was affected with Hypovitaminosis D while the rest of the study subjects exhibited mostly normative in terms of serum vitamin D level. Only a small fraction of study subjects $(n=58)$ representing $0.7 \%$ of the total study-population was suffering from vitamin D toxicity. Among the study subjects $(\mathrm{n}=6469)$ with Hypovitaminosis D, 56.60\% patients $(n=4501)$ were deficient in vitamin $d$ while the rest of the study subjects - $24.70 \%$ were insufficient $(n=1967)$. The frequency distribution of the vitamin D levels (ng/mL) across the study subjects is shown (Figure 1). The distribution analysis revealed a skewed distribution towards the lower levels of vitamin D instead of a Gaussian distribution implying that majority of the study subjects are actually suffering from Hypovitaminosis D. The lowest level of vitamin D was recorded $(4.12 \mathrm{ng} / \mathrm{ml})$ for a female patient of 37 year age whereas the highest level of vitamin D was observed $(102 \mathrm{ng} / \mathrm{ml})$ for another female patient of 48 year. The histogram also shows the number of study subjects that belonged to a particular group.

However, the predicted occurrence was biased due to the over representation of the Hypovitaminosis D patients, as the study-subjects were tested for vitamin-D levels due to clinical symptoms. Therefore to overcome this bias and to estimate the true population-wide prevalence of hypovitaminosis D, a normalization method was performed. By taking into account the total number of subjects $(n=35000)$ that visited the diagnostic center during the same period as the current study (July 2017June 2018) we were able to estimate the natural prevalence of population-wide hypovitaminosis D. This normalization yielded the natural prevalence of population-wide hypovitaminosis D in Bangladesh 
is around 18\% among which Vitamin D deficiency superseded insufficiency. Altogether these findings revealed an interesting scenario in Bangladesh where the severe form of Hypovitaminosis D (deficiency) patients is far more predominant than less severe form (insufficiency) in Bangaldesh.

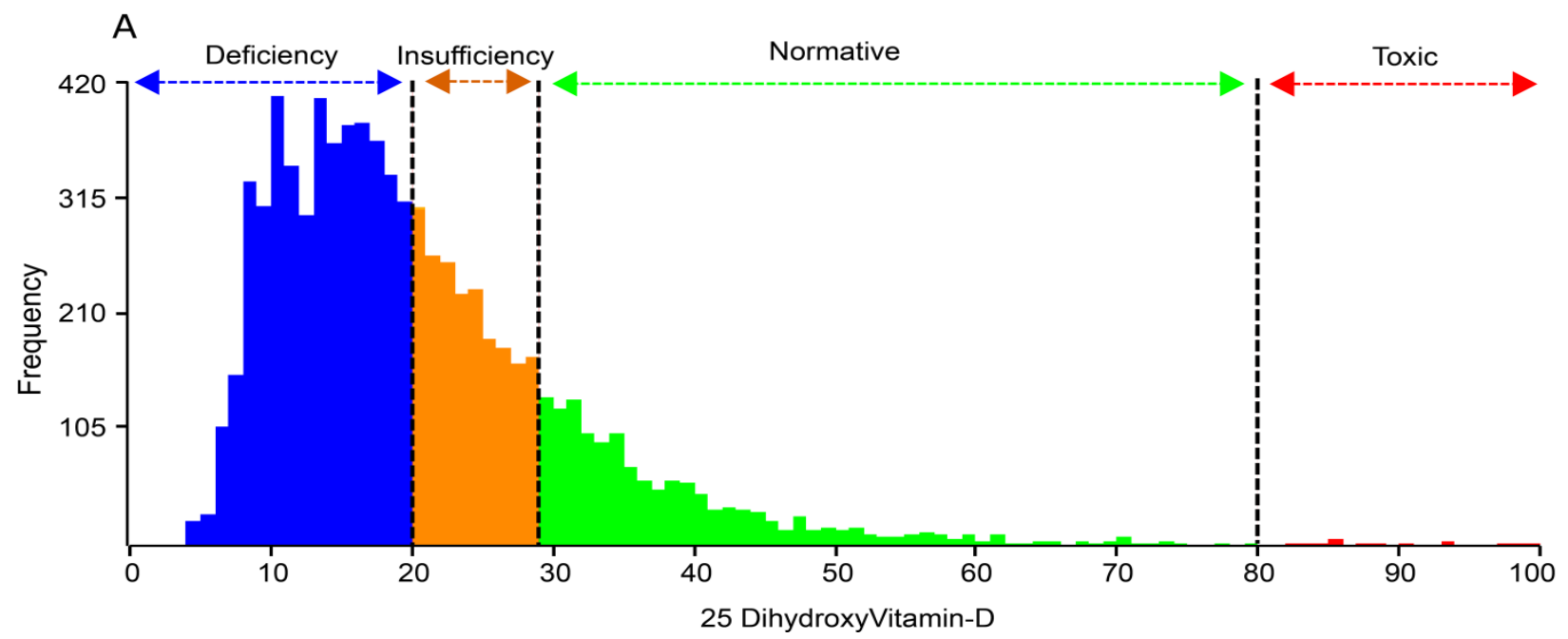

Figure1. The frequency distribution of the vitamin D levels $(\mathrm{ng} / \mathrm{mL})$ across the study subjects

The Impact of Age on the Vitamin D Levels of the Study Population

Since age may play a major role in vitamin D levels and old age is associated with Hypovitaminosis D, we sought to investigate the correlation of the age of study subjects and their vitamin D levels. The frequency distribution analysis of the age of the study subjects showed resemblance to a Gaussian distribution pattern as expected (Figure 2). When the vitamin D levels based clinical stratifications (deficient, insufficient, normative and toxic) of the study subjects were superimposed onto the age histograms, it revealed the predominance of Vitamin
D deficiency compared of the study subjects with age from 20 to 60 . The other age groups, such as below 25 and over 60 exhibited a more uniform pattern where the fraction study subjects with deficiency and insufficiency did not significant vary. Interestingly, four children with the age of 1 year showed vitamin D toxicity. Taken together all these results, it can be concluded that an inverse correlation of vitamin D levels and age was not observed in the current study subjects rather a subgroup of the study subjects with age range of 20 to 60 was found to be more vulnerable to Hypovitaminosis D. Intriguingly, the subgroup of the study subjects with age greater than 60 were less affected with Hypovitaminosis D.

B

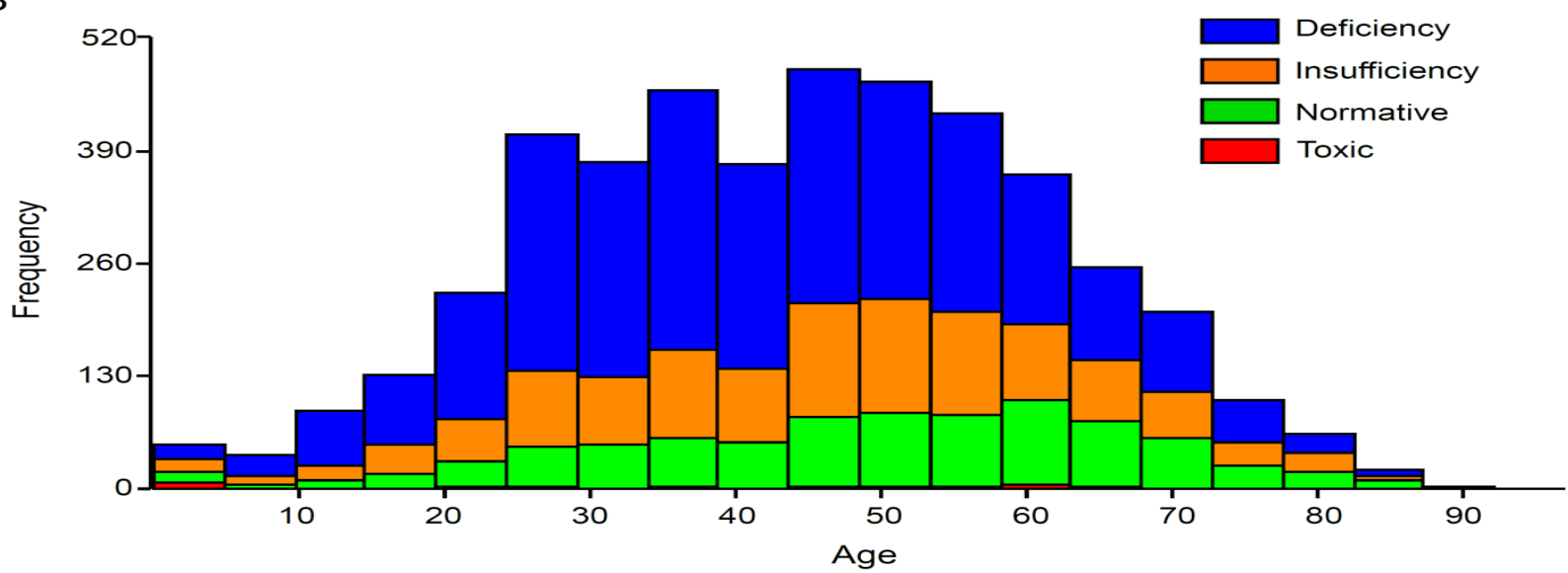

Figure2. The impact of age on the vitamin D levels of the study population 
Patient Risk Stratification and Demographic Profile based on Serum Vitamin-D Levels: A Cross-Sectional Observational Study

The Impact of Gender on the Vitamin D Levels of the Study Population

Next, to determine the impact of the gender of the study subjects on the vitamin D levels, we classified the study subjects according to their gender in different clinically stratified groups such as Vitmain D deficient, insufficient, normative and toxic (Figure 3). The gender-wise classification showed that in all four groups females are predominant than males (Figure 3). In deficient group the percentage of female $(78 \%)$ was far greater than male $(22 \%)$. The gap
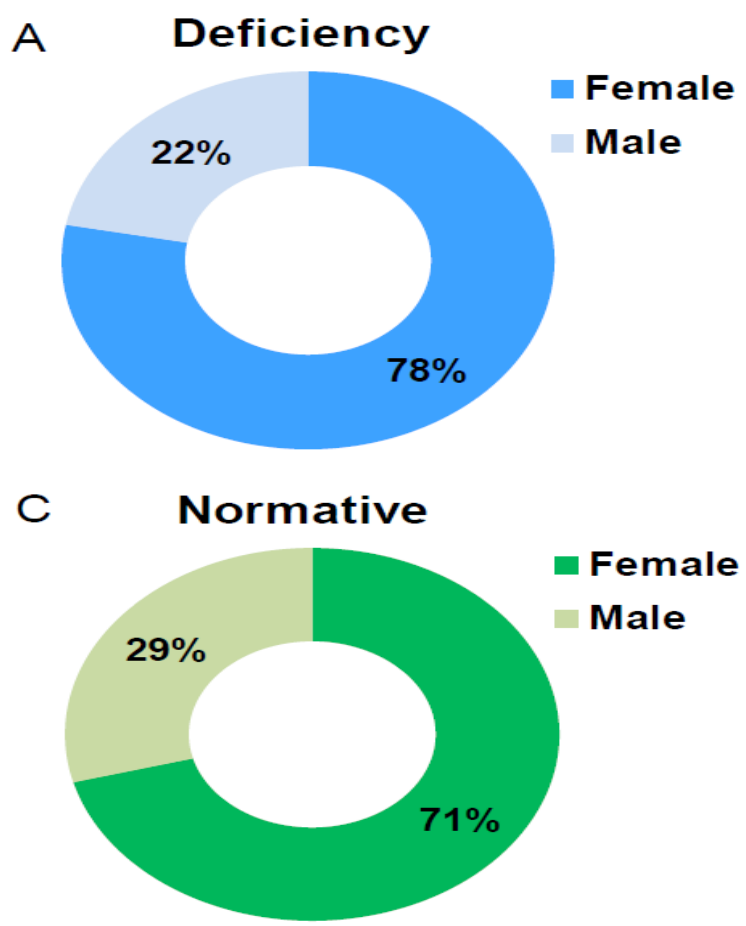

Figure3. The impact of gender on the vitamin D levels of the study population

Analysis of Female Age Groups to Determine the Age-Specific Female Vulnerability towards Vitamin D Deficiency

Having divided the study population according gender, next we wanted to divide the gender-specific study population (female and male) into pre-defined age groups with year age interval and investigate the percentage of the four clinically scarified study subjects in these age groups. To achieve this, the ages of male and female study subjects ranging from 1 to above 80 were divided into nine groups with age interval of 10 year. Interestingly, the age-group analysis between females and males showed some deviations. For males Vitamin D deficiency fraction between females and males were little bit lower but still significant in insufficient (73\% female and 27\% male), normative $(71 \%$ female and $29 \%$ male) and toxic group (62\% female and 38\% male) (Figure 3). In other words the highest female to male ratio was found for the deficient group (3.52) followed by the insufficient group (2.70) and normative group (2.44). The lowest female to male ratio was identified for toxic group (1.63). These results highlighted the fact that female population in Bangladesh are at far greater risk of Hypovitaminosis D than males.

\section{B Insufficiency}
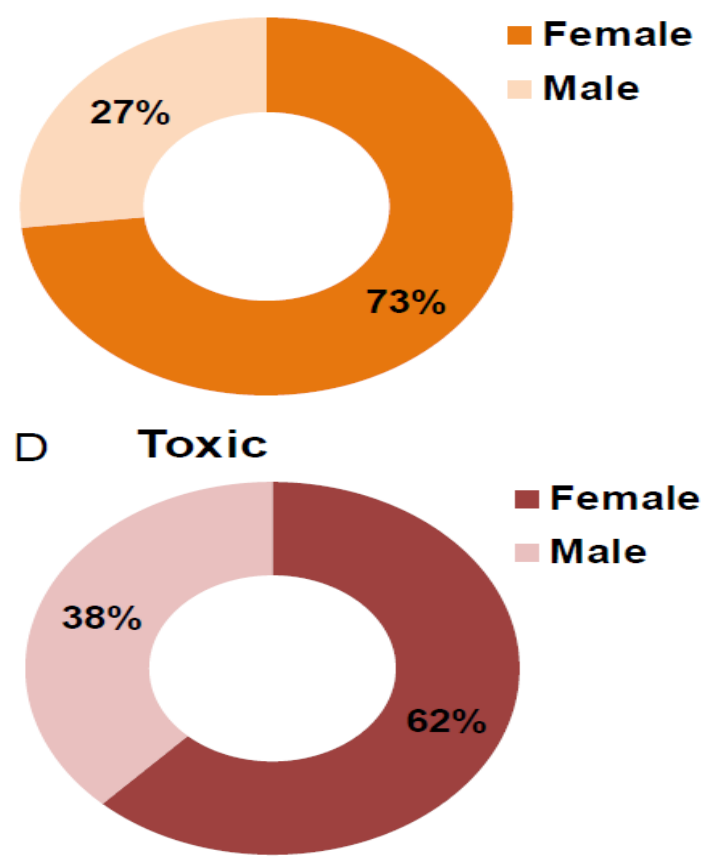


\section{Female}

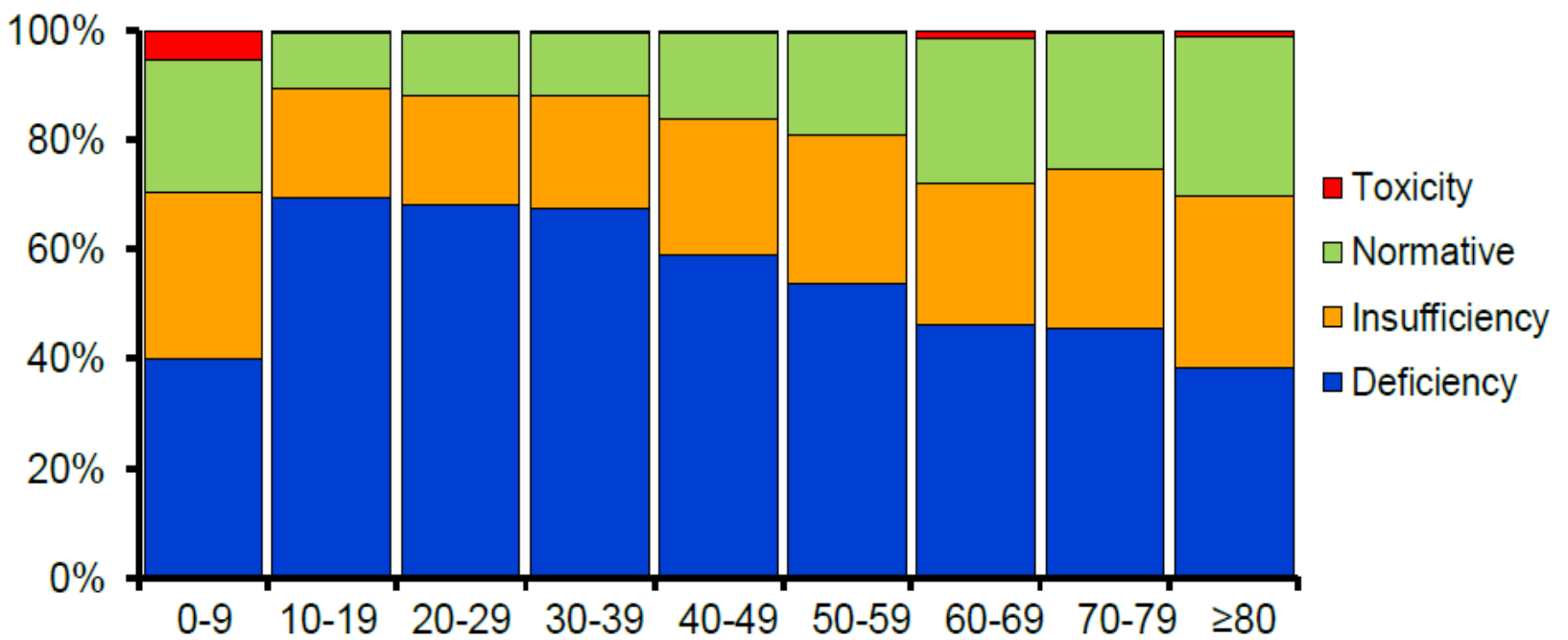

Male

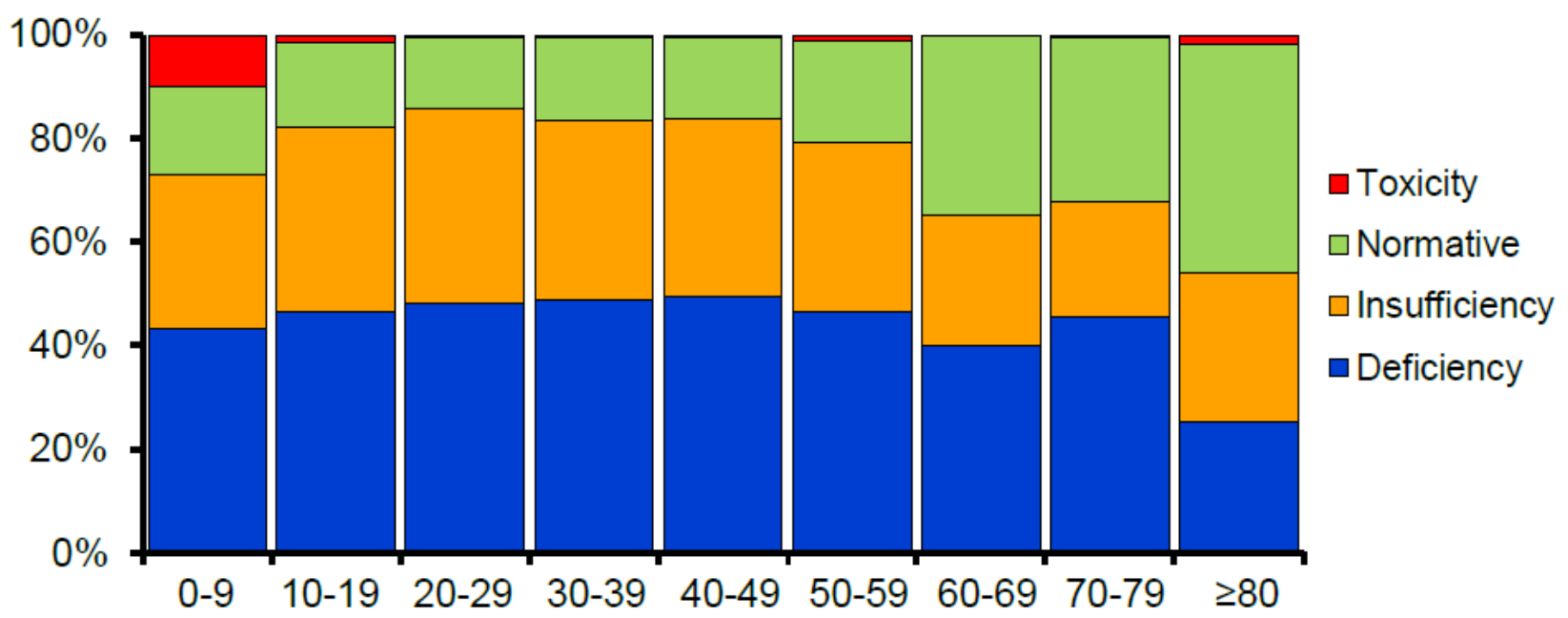

Figure4. Analysis of female age groups to determine the age-specific female vulnerability towards Vitamin $D$ deficiency

\section{DiscuSSION}

Determining vitamin D status of a population can be challenging. It is evident that though vitamin $\mathrm{D}$ deficiency occurs in all parts of the world, significant vitamin D deficiency is very common in all age groups in South Asia and the Middle East ${ }^{7,8}$.In Bangladesh $\left(24^{\circ} \mathrm{N}\right)$, hypovitaminosis $\mathrm{D}$ is more common in women regardless of age, lifestyle, and clothing 9 . Prevalence of hypovitaminosis was 38\% in Bangladeshi women from high-income group and increased slightly to $50 \%$ in women from low income groups ${ }^{9}$. High prevalence of hypovitaminosis D in Bangladesh and in South Asia can be explained by skin pigmentation and traditional clothing. Air pollution and limited outdoor activity further compounds this problem in the urban population.

It is possible that other regions in Asia and Oceania (or even Africa, which remains largely unstudied) may also be similarly affected, but definitive comments cannot be made in absence of more complete data ${ }^{10}$.

The vitamin D status within different European countries shows a high variation. A serum $25(\mathrm{OH})$ D lower than $25 \mathrm{nmol} / \mathrm{l}$ was found in $2 \%$ to $30 \%$ of adults, but this percentage may increase to $75 \%$ or more in older persons in institutions ${ }^{11}$. Despite 
Patient Risk Stratification and Demographic Profile based on Serum Vitamin-D Levels: A Cross-Sectional Observational Study

growing awareness of the multiple health benefits of an adequate vitamin D status, vitamin D insufficiency abounds and is a growing problem in the USA and Canada ${ }^{12}$.

Hypovitaminosis D can affect all age groups, from the newborn to the elderly, and is dependent on several lifestyle and environmental conditions. In general, the cutaneous production of vitamin D3 declines with age ${ }^{13,14}$ and often, the elderly have to be confined to indoors for prolonged periods of time, which compounds the problem. Not surprisingly, therefore, elderly have lower circulating 25(OH)D levels, as compared to the young population from the same regions and with similar skin colour. Similarly, hypovitaminosis D affects both men and women in all age groups but mainly depends on external factors. However, there is evidence that women have lower levels than men in elderly Europeans ${ }^{15}$, in non-institutionalized American civilians of various ethnical subgroups ${ }^{16}$. Moreover, pregnant women ${ }^{17,18}$ and women breast-feeding for long periods of time ${ }^{19}$ are at higher risk of hypovitaminosis D. Gender differences also occur due to clothing differences where adolescent girls and adult women wearing the veil have lower levels of vitamin $D$ throughout the year compared to their male counterparts ${ }^{20}$.

The current study unreveals the higher incidence rate of Vitamin D deficiency among the females of reproductive age. The classical role of vitamin $\mathrm{D}$ was considered in the maintenance of skeletal health in the older adult.

Determination of vitamin D status in serum is based on measurement of the prohormone $25(\mathrm{OH}) \mathrm{D}$ level instead of the active from 1,25(OH)2D due to its stable nature and abundant levels in serum. The severe form of hypovitaminosis known as vitamin D deficiency istypically recognized by the onset of bone associated diseases such as rickets or osteomalacia. These type of bone diseases are associated with the serum $25(\mathrm{OH})$ D level of less than $10 \mathrm{ng} / \mathrm{mL}$. To characterize the less severe form of hypovitaminosis another terminology has been used in recent times which is called Vitamin D insufficiency to indicate the suboptimal levels of serum 25(OH)D. The stratification of patients into vitamin $\mathrm{D}$ deficiency and insufficiency category solely based on the serum 25(OH)D level are biologically meaningful remains to be controversial.
Traditionally, it was assumed that older adults are at risk of vitamin D deficiency resulting from the reduced cutaneous synthesis and dietary intake of vitamin D. Interestingly our study reveals a different scenario in Bangladesh where higher incidence of vitamin D deficiency was marked in female population of reproductive age. This notion vitamin deficiency onset in relatively younger woman was not entirely novel. Previously Woo et al. showed high rates of Hypovitaminosis D was found in females of childbearing age in Beijing and Hong Kong where the authors estimated a higher occurrence of Vitamin D insufficiency than deficiency. In contrast our results showed an opposite trend of hypovitaminosis D in Bangladesh where the rate of occurrence of Vitamin D deficiency is higher than insufficiency. The higher incidence of vitamin D deficiency in comparison to insufficient appears to be very intriguing and demands for further explanation.

Another interesting aspect is the Vitamin-D toxicity (VDT) that was observed in 58 individuals representing $0.7 \%$ of the total study subjects of which 12 subjects have an age of under-five. The adult patients who showed significant higher levels of Vitamin D may have likely undergone a treatment regimen with Vitamin D to compensate their hypovitaminosis and the toxicity may have resulted from the overdose or mal-adjustment of the Vitamin D dosage. Previously it has been shown that VDT canbe stemmed from the inappropriate intake of very high doses of vitamin D and is associated with hypercalcemia. Serum 25hydroxyvitamin D [25(OH)D] concentrations higher than $150 \mathrm{ng} / \mathrm{ml}$ (375 nmol/l) are the hallmark of VDT due to vitamin D overdosing. However, the vitamin D toxicity for children under 5 years of age cannot be explained by the increased intake off Vitamin-D. It has been observed that endogenous VDT may develop from excessive production of an active vitamin D metabolite - 1,25(OH)2D in granulomatous disorders and in some lymphomas or from the reduced degradation of that metabolite in idiopathic infantile hypercalcemia. Endogenous VDT may also develop from an excessive production of 25(OH)D and 1,25(OH)2D in congenital disorders, such as Williams-Beuren syndrome. It would be necessary to further diagnose those subjects to explain their toxic serum Vitamin levels. 
Patient Risk Stratification and Demographic Profile based on Serum Vitamin-D Levels: A Cross-Sectional Observational Study

\section{CONCLUSION}

Numbers of people with VDD are continuously increasing; the importance of this hormone in overall health and the prevention of chronic diseases are at the forefront of research. VDD is very common in all age groups. Very few foods contain vitamin D therefore guidelines recommended supplementation of vitamin D at tolerable UL levels. It is also suggested to measure the serum 25-hydroxyvitamin D level as the initial diagnostic test in patients at risk for deficiency. Treatment with either vitamin $\mathrm{D}_{2}$ or vitamin $\mathrm{D}_{3}$ is recommended for the deficient patients. More research is required to recommend screening individuals who are not at risk for deficiency or to prescribe vitamin $\mathrm{D}$ to attain the noncalcemic benefit for cardiovascular protection.

\section{REFERENCES}

[1] Holick MF. Vitamin D deficiency. N Engl J Med. 2007;357:266-81.

[2] Nair R, Maseeh A. Vitamin D: The "sunshine" vitamin. J PharmacolPharmacother. 2012 AprJun; 3(2): 118-126. PMID: 22629085.

[3] Alves C. Diagnosis and Treatment of Hypovitaminosis D: Recommendations from India and Brazil. Indian J EndocrinolMetab. 2017 May-Jun; 21(3): 367-368. PMID: 28553587.

[4] Hollis BW,Wagner CL. Normal serum vitamin D levels. N Engl J Med. 2005; 352(5):515-6.

[5] Endocrine Society of India Expert Group. Vitamin D deficiency in India: Recommendations for prevention and treatment. Indian $\mathrm{J}$ EndocrinolMetab. 2016 In Press.

[6] Maeda SS, Borba VZ, Camargo MB, Silva DM, Borges JL, Bandeira F, et al. Brazilian Society of Endocrinology and Metabology (SBEM). Recommendations of the Brazilian Society of Endocrinology and Metabology (SBEM) for the diagnosis and treatment of hypovitaminosis D. Arq Bras EndocrinolMetabol. 2014;58:411-33. PMID: 25166032

[7] Weng FL, Shults J, Leonard MB, Stallings VA, Zemel BS (2007) Risk factors for low serum 25hydroxyvitamin D concentrations in otherwise healthy children and adolescents. Am J ClinNutr 86: 150-158.

[8] Mithal A, Wahl DA, Bonjour JP, Burckhardt P, Dawson-Hughes B, Eisman JA, et al. (2009) Global vitamin D status and determinants of hypovitaminosis D. OsteoporosInt 20: 18071820.

[9] Islam MZ, Akhtaruzzaman M, Lamberg-Allardt C (2006).Hypovitaminosis D is common in both veiled and nonveiled Bangladeshi women. Asia Pac J ClinNutr 15:81-87

[10] Islam MZ, Lamberg-Allardt C, Karkkainen M et al (2002). Vitamin D deficiency: a concern in premenopausal Bangladeshi women of two socio-economic groups in rural and urban region. Eur J ClinNutr 56:51-56.

[11] Lips P (2001) Vitamin D deficiency and secondary hyperparathyroidism in the elderly consequences for bone loss and fractures and therapeutic implications. Endocr Rev 22:477-501

[12] Vieth R, Cole DE, Hawker GA et al (2001) Wintertime vitamin D insufficiency is common in young Canadian women, and their vitamin D intake does not prevent it. Eur J ClinNutr 55:1091-1097

[13] Holick MF, Matsuoka LY, Wortsman J (1989) Age, vitamin $\mathrm{D}$, and solar ultraviolent (letter). Lancet 2:1104-1105.

[14] Maclaughlin J, Holick MF (1985) Aging decreases the capacity of human skin to produce vitamin D3. J Clin Invest 76:1536-1538.

[15] Vanderwielen RPJ, Lowik MRH, Vandenberg H et al (1995) Serum vitamin D concentrations among elderly people in Europe. Lancet 346:207-210

[16] Looker AC, Dawson-Hughes B, Calvo MS et al (2002) Serum 25-hydroxyvitamin D status of adolescents and adults in two seasonal subpopulations from NHANES III. Bone 30:771777.

[17] Judkins A, Eagleton C (2006) Vitamin D deficiency in pregnant New Zealand women. N Z Med J 119:U2144

[18] Bowyer L, Catling-Paull C, Diamond T et al (2008) 
Patient Risk Stratification and Demographic Profile based on Serum Vitamin-D Levels: A Cross-Sectional Observational Study

Vitamin D, parathyroid hormone and calcium levels in pregnant women and their neonates. ClinEndocrinol (Oxf) .

[19] Challa A, Ntourntoufi A, Cholevas V et al (2005) Breastfeeding and vitamin D status in Greece during the first 6 months of life. Eur J Pediatr 164:724-729

[20] Mishal AA (2001) Effects of different dress styles on vitamin D levels in healthy young Jordanian women. OsteoporosInt 12:931-935.

Citation: Mousumi Sanyal, Richmond Ronald Gomes, Yearul Kabir, Sajib Chakraborty. Patient Risk Stratification and Demographic Profile based on Serum Vitamin-D Levels: A Cross-Sectional Observational Study. Open Access Journal of Internal Medicine. 2020; 3(2): 10-17.

Copyright: (C) 2020 Mousumi Sanyal, Richmond Ronald Gomes, Yearul Kabir, Sajib Chakraborty. This is an open access article distributed under the Creative Commons Attribution License, which permits unrestricted use, distribution, and reproduction in any medium, provided the original work is properly cited. 